\title{
Active straight leg raising (ASLR) competence improves with reverse-ASLR exercises and not repeating ASLR exercises
}

\author{
Hiroshi Takasaki*, Shota Kawazoe \\ Department of Physical Therapy, Saitama Prefectural University, Koshigaya, Japan
}

In the Functional Movement Screen (FMS), a subgroup of those with a score of 1 due to limitations in the active straight leg raising (ASLR) but not in the passive straight leg raising is considered to have a stability or motor control dysfunction (SMCD). The FMS proposes the use of the movements in a reverse pattern to improve FMS scores. The aim of this study was to investigate whether the reverse pattern of the ASLR (reverse-ASLR) was more effective than repeating the ASLR to improve the FMS score in participants with the FMS ASLR score of 1 due to the SMCD (ASLR-1-SMCD). A two-armed randomized controlled trial was conducted in individuals with the ASLR-1-SMCD. The intervention was either the reverse-ASLR or the ASLR exercise on both sides at home for a month followed by a 1-month wait-and-see interval, wherein the primary outcome measure was the right FMS ASLR score. Forty partici- pants were randomized to the ASLR exercise group $(n=20)$ or the reverse-ASLR exercise group $(n=20)$. The Fisher exact test demonstrated a statistically significant difference $(P=0.020)$ in the proportion of those with FMS ASLR score improvement to a score of 2 (ASLR exercise group, one; reverse-ASLR exercise group, eight) at follow-up 1, but no significance $(P=0.106)$ at follow-up 2 (ASLR exercise group, none; reverseASLR exercise group, four). This study indicated that the reverse-ASLR exercise was more effective than repeating the ASLR exercise in order to improve the ASLR score among individuals with the ASLR-1-SMCD.

Keywords: Corrective exercises, Functional Movement Screen, Reverse patterning

\section{INTRODUCTION}

Active straight leg raising (ASLR) is a common test for multisegmental control in the trunk and lower limbs (Bennett et al., 2017) and is included in the Functional Movement Screen (FMS) (Cook, 2010). The FMS is a valid and reliable system for grading movement competency with 4 grades (score $0-3$ ) in each of the seven screening tests (Moran et al., 2016; Warren et al., 2018). Briefly, scores of 1, 2, and 3 indicate impaired, normal, and good movement competencies in individuals without pain during the movement, respectively (Cook, 2010). The FMS ASLR scores of 1-3 are determined based on the location of a vertical line through the malleolus of the elevated lower limb on the remaining lower limb that stays still (score of 1 , below the knee joint line of the remaining lower limb; score of 2, between the mid-thigh and the knee joint line of the remaining lower limb; and score of 3, above the mid-thigh of the remaining lower limb). Moreover, individuals with the FMS ASLR score of 1 were divided into two subgroups: (a) those with limitations in both ASLR and passive straight leg raising and (b) those with limitation in the ASLR but not in the passive straight leg raising. An underlying mechanism of such a limited ASLR was assumed to be in deficits in structural flexibility in the former group and in stability or motor control dysfunction (SMCD) of the trunk and hip muscles in the latter group (ASLR-1-SMCD group) (Cook, 2010).

The FMS proposes corrective exercises to improve FMS scores (Cook, 2010). The corrective exercises are classified into four types, and one of them is the reverse patterning (Cook, 2010). In fact,
*Corresponding author: Hiroshi Takasaki (iD https://orcid.org/0000-0003-3699-2294 Department of Physical Therapy, Saitama Prefectural University, Sannomiya 820, Koshigaya, Saitama 343-8540, Japan

Email: physical.therapy.takasaki@gmail.com

Received: December 24, 2020 / Accepted: January 28, 2021
This is an Open Access article distributed under the terms of the Creative Commons Attribution Non-Commercial License (https://creativecommons.org/licenses/by-nc/4.0/) which permits unrestricted non-commercial use, distribution, and reproduction in any medium, provided the original work is properly cited. 
exercises using the reverse patterning have been included in a cohort study of athletes that showed FMS score improvement (Tejani et al., 2019). Conversely, physicians may prescribe patients and clients exercises which involve repeating the movement that they cannot conduct perfectly in order to improve the competency of the movement. As far as the authors know, direct evidence supporting the effectiveness of the reverse patterning of the ASLR is lacking.

Furthermore, knowledge about the underlying mechanisms of the SMCD is limited. One promising mechanism includes impaired feedforward control, which is supported by a previous study showing the difference in relative latency of the onsets of the right internal oblique muscle and the left gluteus maximum muscle to the onset of the right rectus femoris muscle during the right ASLR between individuals with ASLR-1-SMCD and those with the FMS ASLR score of 3 (Takasaki et al., 2020). Another promising mechanism may include impaired sensorimotor control due to impaired proprioception with or without impaired motor control because plasticity in somatosensory function occurred with motor learning (Mirdamadi and Block, 2020; Ostry and Gribble, 2016; Ostry et al., 2010; Wong et al., 2011).

This randomized controlled trial primarily aimed to investigate whether the reverse patterning of the ASLR was more effective to improve the FMS score than the exercise by repeating the ASLR in individuals with the ASLR-1-SMCD. As the secondary aim, this study also preliminarily investigates differences in relative latency of the onset of the abdominal and hip muscles to the onset of the rectus femoris muscle during the ASLR and measures of the sensorimotor control in the trunk and hip.

\section{MATERIALS AND METHODS}

\section{Design}

This study was a two-armed randomized controlled trial of 1-month interventions: the reverse patterning of the ASLR (reverse-ASLR exercise) and the ASLR exercise.

\section{Participants}

Participants were recruited using convenience sampling through advertisements in the Saitama Prefectural University from July 2018 to January 2019. Inclusion criteria were based on a previous study (Takasaki et al., 2020), which included (a) age of > 17 years, (b) no pain in the spine or lower extremities during the previous month, (c) no history of diagnosed spinal deformities or central nervous system disorders, and (d) ASLR-1-SMCD on both sides of
ASLR. The participants were selected by an author certified for FMS level 1 (HT).

The study methodology was approved by the human research ethics committee in the Saitama Prefectural University (No. 30006) and was preregistered in a clinical trial registration (UMIN000033277). A written informed consent was obtained from each participant prior to data collection.

\section{Interventions}

Fig. 1 illustrates the procedures of the reverse-ASLR and ASLR exercises. In the reverse-ASLR exercise, the participants raised their lower limbs symmetrically as high as possible (up to $90^{\circ}$ hip flexion), with extended knees extended as the starting position. Subsequently, the participants slowly lowered one lower limb to $0^{\circ}$ hip flexion with their knee extended while the other lower limb stationary at the starting position. The participants were instructed to perform this movement for 3 sec in a normal breathing pattern. Attention was given to holding the lower limb stationary while smoothly lowering the opposite lower limb. Finally, the participants bent the hip and knee of the lower limb and then extended the knee back to the starting position. In the ASLR exercise, the start position was supine. The participants undertook ASLR as high as possible, reaching the end range in $3 \mathrm{sec}$ in a normal breathing pattern. Attention was given to holding the lower limb stationary while smoothly raising the opposite lower limb. Finally, the participants bent the knee and extended the hip of the raised lower limbs to the starting position. The participants performed either the reverse-ASLR or the ASLR exercise on both sides at home for one month. A set of the exercise included 10 repetitions of the movements, and the participants were asked to perform three sets of the exercise per day. The time of the exercise was not controlled. The participants were also asked to record the completion of each set in an exercise diary (Nishimoto and Takasaki, 2019; Takasaki et al., 2018). Each participant practiced the allocated exercise with real-time feedback from an author (HT) at the end of the baseline assessment and performed the home exercise with reference to the exercise chart (Fig. 1).

\section{The primary outcome measure}

The primary outcome measure was the right FMS ASLR score as rated by an examiner certified for FMS levels 1-2 using a standardized instruction (Cook, 2010). The participants performed the right ASLR 3 times, and the highest score was recorded (Cook, 2010). The examiner was blinded to the participant's allocation to the interventions throughout the study. 


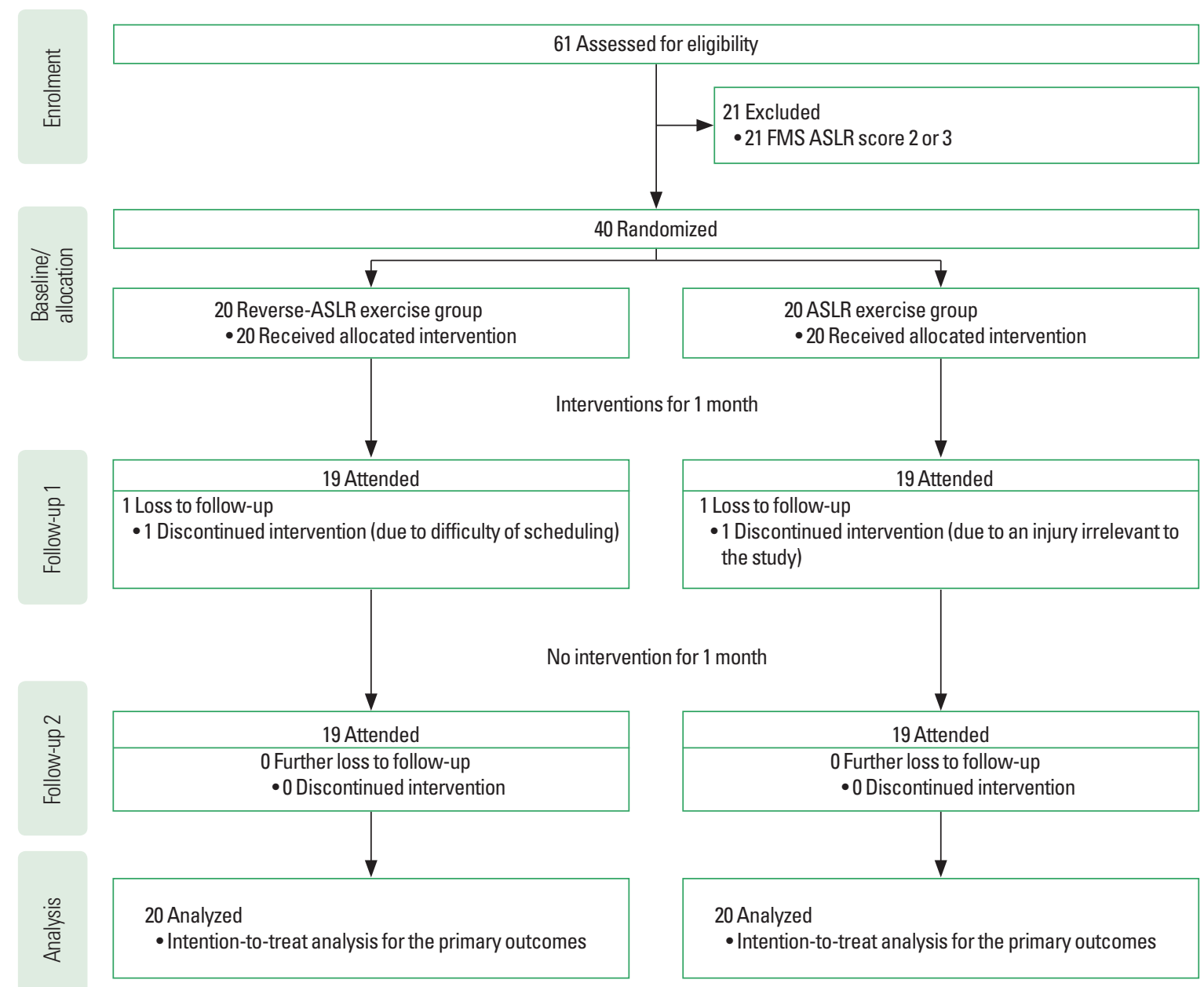

Fig. 1. Active straight leg raising (ASLR) and reverse-ASLR exercises. Exercises for the right side are presented. Time for lowering of the leg was about 3 sec in the reverse-ASLR exercise. Time for raising of the leg was about $3 \mathrm{sec}$ in the ASLR group. ASLR, active straight leg raising.

\section{The secondary outcome measures}

The measures of the feedforward control included the following: (a) relative latency of the onsets of the left rectus abdominis muscle, bilateral internal oblique muscles, bilateral external oblique muscles, left gluteus maximum muscle, and left hamstring muscles to the onset of the right rectus femoris muscle during the right ASLR and (b) amplitude of activity in the left rectus abdominis muscle, bilateral internal oblique muscles, bilateral external oblique muscles, left gluteus maximum muscle, and left hamstring muscles at an early phase of the ASLR. Surface electromyography was used for both measures and the method of data collection and analysis were followed to the previous studies (Takasaki and Okubo, 2020; Takasaki et al., 2020). The measures of the sensorimotor control included the following: (a) 2-point discrimination test over the lower back as a measure of proprioception and (b) active repo- sitioning test of the right hip as a measure of proprioception with motor control.

Regarding the measures of relative latency, the electromyography onset of each muscle was manually identified using raw electromyography signals, which has high inter-session reliability (Hodges and Bui, 1996). To maintain assessor blinding, a research assistant randomized the order of electromyography data presentation and masked the labels of electromyography data during analyses to the examiner. The examiner identified the electromyography onset using the following criteria: (a) increased electromyography amplitude above baseline levels, (b) recruitment of additional motor units, or (c) increased firing rate of active motor units. For the amplitude of muscle activity, the amplitude data during $50 \mathrm{~ms}$ after the onset of rectus femoris muscle activity were calculated. The mean of the first 10-15 datasets was used for statistical anal- 
yses of both secondary measures (Takasaki et al., 2020; Takasaki and Okubo, 2020).

For the sensorimotor control measures, the 2-point discrimination test was reported to have the greatest level of discriminant validity between individuals with and without low back pain among sensorimotor control measures (Ehrenbrusthoff et al., 2018). The 2-point discrimination test is assumed to offer a clinical signature of primary somatosensory cortex representation (Lotze and Moseley, 2007). The examiner was blinded to the assignment of the interventions and data from prior examinations. This study followed an established procedure with high inter-session and inter-examiner reliability (Ehrenbrusthoff et al., 2016). Briefly, participants were placed in a prone position with their backs exposed. The measurement tool was a plastic caliper ruler with a $1-\mathrm{mm}$ precision for evaluation. The caliper ruler was lightly placed perpendicular to the lumbar spine, and the center of the caliper ruler was on the transverse process of $\mathrm{L} 5$. The measurement side was randomized. Five datasets were collected, and the last four datasets were averaged for the analysis. Testing was conducted in an ascending manner by incrementing the caliper distance of $5 \mathrm{~mm}$ from $20 \mathrm{~mm}$. The time between each increment was no more than a few seconds. Catch trials were also included every 3-7 measurement. The caliper distance where the participant first identified 2 points was noted. When the participant again identified 2 points with a caliper distance incremented by $5 \mathrm{~mm}$, the result was recorded. Moreover, when the participant did not identify the stimulus as two separate points, the examiner continued the testing by incrementing the caliper distance until two consecutive correct identifications were identified by the participant.

Improved joint repositioning acuity has been reported in some randomized controlled trials including motor control training for individuals with spine disorders (Jull et al., 2007; Wattananon et al., 2019). Therefore, a measure of active joint repositioning acuity of the hip with two inertial measurement unit sensors attached on the pelvis and the lateral thigh of the right lower limb was included. The examiner was blinded to the assignment of the interventions and data from prior examinations. This study followed an established procedure with high inter-session reliability (Arvin et al., 2015). Briefly, participants were positioned in a one-leg standing position on their left leg on a 10-cm-high block with their right leg unsupported and aligned with the left leg. The participant was blindfolded and allowed to touch the bar beside them with both hands. First, the participants actively abducted their right hip with the knee straight to a target angle, $10^{\circ}-40^{\circ}$ abduction. The participants memorized the target angle for $4 \mathrm{sec}$ and then returned to the start position and maintained the position for 3 sec. Subsequently, the participant actively repositioned their right leg to the target position as accurate as possible within 5 sec and pressed a button with their hand. Four datasets were collected, and absolute errors were averaged for the analysis.

The Japanese version of Euro QoL 5 Dimensions (EQ-5D) (Shiroiwa et al., 2016) was assessed for quality of life. Time for reaching to $95 \%$ of the hip flexion range was also calculated from the two inertial measurement unit sensors during the evaluation of the electromyography measures with ASLR tasks. At the baseline assessment, data of demographics including age, sex, and body mass index were also evaluated.

\section{Data acquisition procedures}

After acquisition of the EQ-5D, the FMS score was assessed followed by the 2-point discrimination test. Subsequently, the active joint repositioning acuity of the hip was assessed. The assessments with electromyography and motion analysis were then conducted by repeating the ASLR based on an established procedure (Takasaki and Okubo, 2020; Takasaki et al., 2020). Briefly, the participants placed their arms beside the trunk with palms facing up. The participants elevated their right lower limb to their end range during hip flexion for $1 \mathrm{sec}$ immediately after they saw the lighting placed in front of their face from the relaxed supine lying position while keeping the knee straight. The timing of lighting was randomized, ranging from $10 \mathrm{sec}$ to $15 \mathrm{sec}$. The participants repeated ASLR 17-23 times until at least 10 clear electromyography datasets were obtained. Finally, three sets of maximum voluntary contraction (MVC) for $3 \mathrm{sec}$ were conducted in each muscle according to the standard procedures, where the order of MVC was consistent throughout the study.

\section{Electromyography data processing}

Self-adhesive $\mathrm{Ag} / \mathrm{AgCl}$ electrodes (electrocardiographic electrodes 2009111-150, CareFusion, San Diego, CA, USA) were attached on standardized positions of the left rectus abdominis muscle, bilateral internal oblique muscles, bilateral external oblique muscles, left gluteus maximum muscle, right rectus femoris muscle, and left hamstring muscles with a 20-mm interelectrode distance (Takasaki and Okubo, 2020; Takasaki et al., 2020). For the hamstring muscle, the electrode was placed parallel to the muscle in the center of the back of the thigh (Criswell, 2011).

Electromyography data were measured using a myoMUSCLEsystem (TELEmyo DTS 580-8M, NORAXON USA, Scottsdale, AZ, USA) with a sampling frequency of $1,500 \mathrm{~Hz}$. The amplitude of 
muscle activity was calculated by reducing the electrocardiographic complex, filtering the electromyography data using a $20-500 \mathrm{~Hz}$ band-pass filter, and calculating the root mean square using a 100msec sliding window. The amplitude of electromyography data was presented with \%MVC.

\section{Motion data processing}

Motion data were captured with the two inertial measurement unit sensors on the pelvis and the right thigh with a sampling frequency of $100 \mathrm{~Hz}$. Data were processed using myoMOTIONsystem (EM-M07, NORAXON USA) that was synchronized in the electromyography system and an external trigger of a light.

\section{Randomization}

Randomization was performed using sealed opaque envelopes. Concealed allocation was maintained as patients selected an envelope where the intervention group was written. Patients were asked not to reveal their intervention group to an examiner throughout the study.

\section{Sample size estimation}

Sample size estimation was performed in a pilot study with the first 20 participants (i.e., 10 participants in each group), which was recommended by Sandvik et al. (1996). In the pilot study, six participants in the reverse-ASLR exercise group (60\%) and one participant in the ASLR exercise group (10\%) had FMS score improvement to a score of 2 at follow-up 1. G*Power 3 (Faul et al., 2007) estimated that 34 participants (i.e., 17 participants in each group) were required, considering $\alpha$ value of $0.05, \beta$ value of 0.2 , and proportions of 0.6 and 0.1 in the Fisher exact test. Considering the $20 \%$ dropout, 40 patients (i.e., 20 participants in each group) were estimated in the formal study. The methodology did not change after the pilot study; thus, recruitment of additional 20 participants was continued for the formal study.

\section{Statistical analyses}

IBM SPSS Statistics ver. 21.0 (IBM Co., Armonk, NY, USA) was used for statistical analyses. The intention-to-treat analysis was used for the primary outcomes, where a value at the baseline assessment was used at the follow-up assessments for the droppedout participants. The two-tailed Fisher exact test was used for the primary outcome measure at each follow-up, and the effect size of Cohen $\mathrm{h}$ was calculated, where the following interpretation was performed: $0.2,0.5$, and 0.8 for small, medium, and large effect size, respectively. For convenience, the two-way repeated measures analysis of variance (ANOVA) was used for the secondary measures of the EQ-5D, measures of the 2-point discrimination test, hip abduction active repositioning acuity, and electromyography, comparing the effects between the reverse-ASLR and the ASLR exercise group. Furthermore, an interaction effect was assessed for the measures of the 2-point discrimination test, hip abduction active repositioning acuity, and electromyography between those with and without FMS ASLR score improvement at follow-up 1 using the two-way repeated measures ANOVA with data at baseline and follow-up 1. In order to confirm consistency of the ASLR tasks between groups and time points, time for reaching $95 \%$ of the hip flexion range was assessed using the repeated measures ANOVA at each group and the $t$-test between the groups at each time point. In order to confirm consistency of the exercise compliance, the compliance converted into \% was compared between the groups using the $t$-test. The level of $\alpha$ was set at $5 \%$. For ANOVAs, the Greenhouse-Geisser correction was used when the Mauchly test of sphericity was statistically significant.

\section{RESULTS}

\section{Results of the participants}

Electromyography data of the hamstring muscle for one participant in the ASLR exercise group at follow-up 1 were missed due to a technical problem; thus, data imputation was performed with the patient's baseline data. One participant in each group droppedout before follow-up 1 (Fig. 2). No adverse event was observed caused by participating in this study. Data imputation with baseline data was performed for follow-ups 1 and 2 for measurement outcomes. Table 1 presents the demographics of each group.

\section{The primary outcome}

Regarding the primary outcomes, one participant in the ASLR exercise group had improvements of the FMS score to 2, and eight participants in the reverse-ASLR exercise group had improvements of the FMS score to 2 at follow-up 1. The two-tailed Fisher exact test demonstrated $P=0.020$ with $b=1.12$, indicating a large effect size. At follow-up 2, nobody in the ASLR exercise group had the FMS score of 2, and four out of the eight participants with the FMS score improvement at follow-up 1 maintained the FMS score of 2. The two-tailed Fisher exact test demonstrated $P=0.106$ with $h=1.68$, indicating a large effect size.

\section{The secondary outcomes}

Fig. 3 presents the relative latency in the reverse-ASLR exercise 
Reverse-ASLR exercise
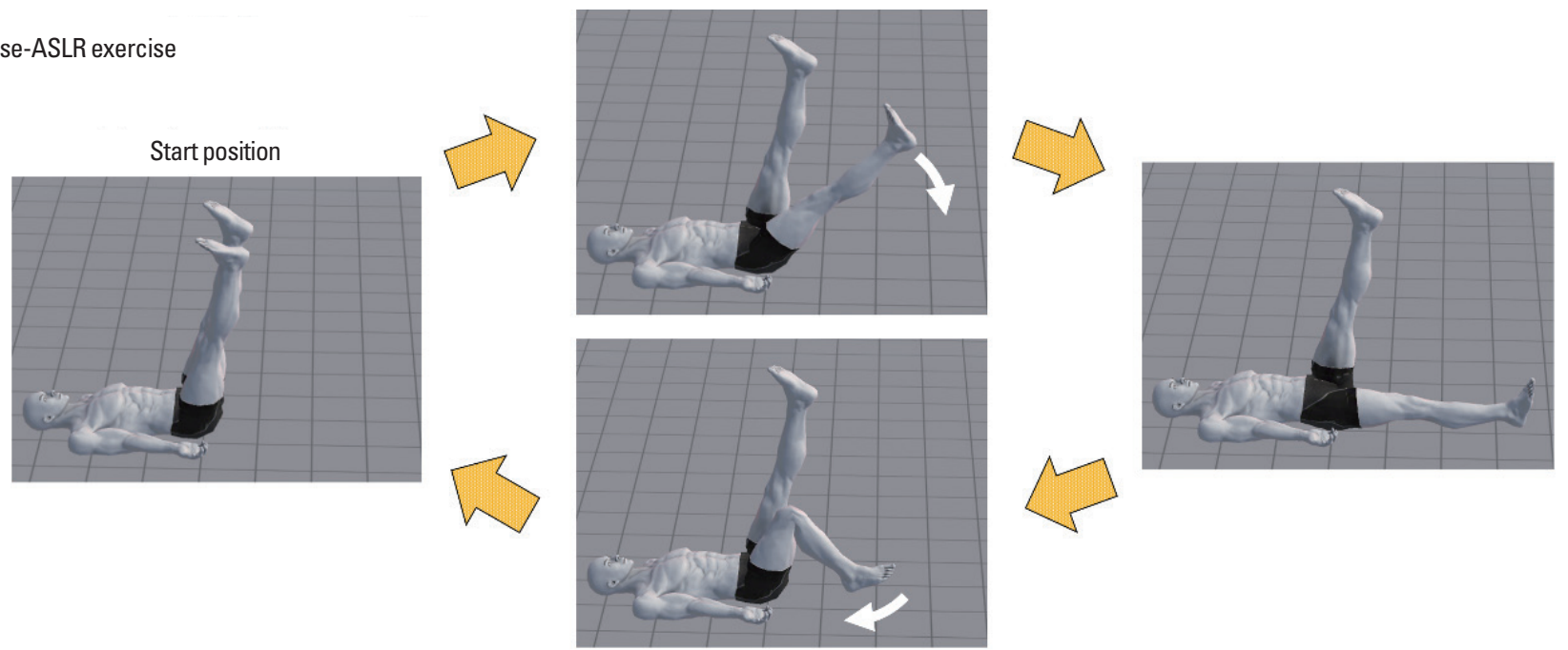

ASLR exercise

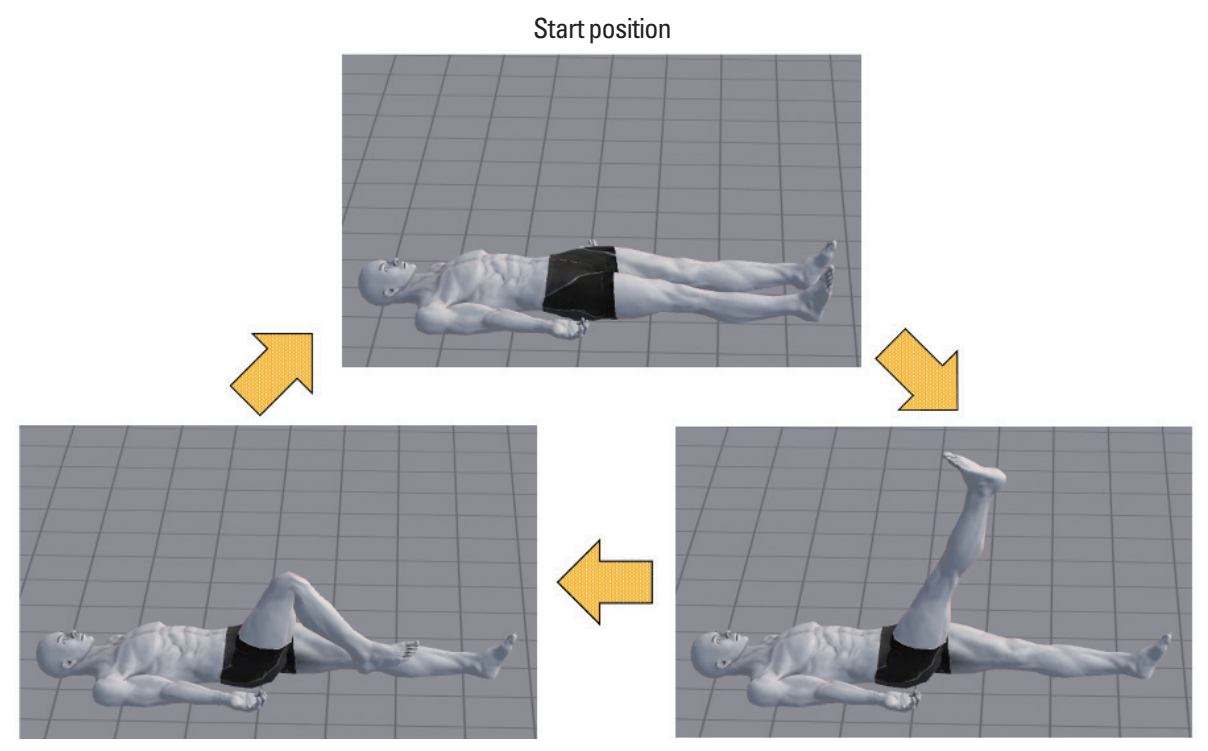

Fig. 2. Flow of the participants. ASLR, active straight leg raising.

Table 1. Demographics of the two groups

\begin{tabular}{lcc} 
Variable & $\begin{array}{c}\text { ASLR exercise group } \\
(\mathrm{n}=20)\end{array}$ & $\begin{array}{c}\text { Reverse-ASLR exercise } \\
\text { group }(\mathrm{n}=20)\end{array}$ \\
\hline Age $(\mathrm{yr})$ & $21.95 \pm 4.90$ & $23.60 \pm 6.89$ \\
Gender, male:female $(\mathrm{n})$ & $8: 12$ & $12: 8$ \\
Body mass index $\left(\mathrm{kg} / \mathrm{m}^{2}\right)$ & $21.83 \pm 2.45$ & $21.53 \pm 2.76$ \\
\hline
\end{tabular}

Values are presented as mean \pm standard deviation or number. ASLR, active straight leg raising.

group and the ASLR exercise group, where a statistically significant interaction effect was not seen in any muscle $(P>0.05)$. Fig. 4 presents the amplitude of muscular activity at an early phase of
ASLR in the reverse-ASLR exercise group and the ASLR exercise group, where a statistically significant interaction effect was not seen in any muscle $(P>0.05)$. Table 2 presents data of the secondary outcomes at each time point except the secondary outcomes using the electromyography and time for reaching up to $95 \%$ of the hip flexion range. No variable was found with a statistically significant interaction effect $(P>0.05)$.

As post boc, changes in the measures of the 2-point discrimination test, hip abduction active repositioning acuity, and electromyography between baseline and follow-up 1 were also compared between individuals with and without FMS ASLR score improvement at follow-up 1 using the $t$-test. Table 3 presents the interac- 

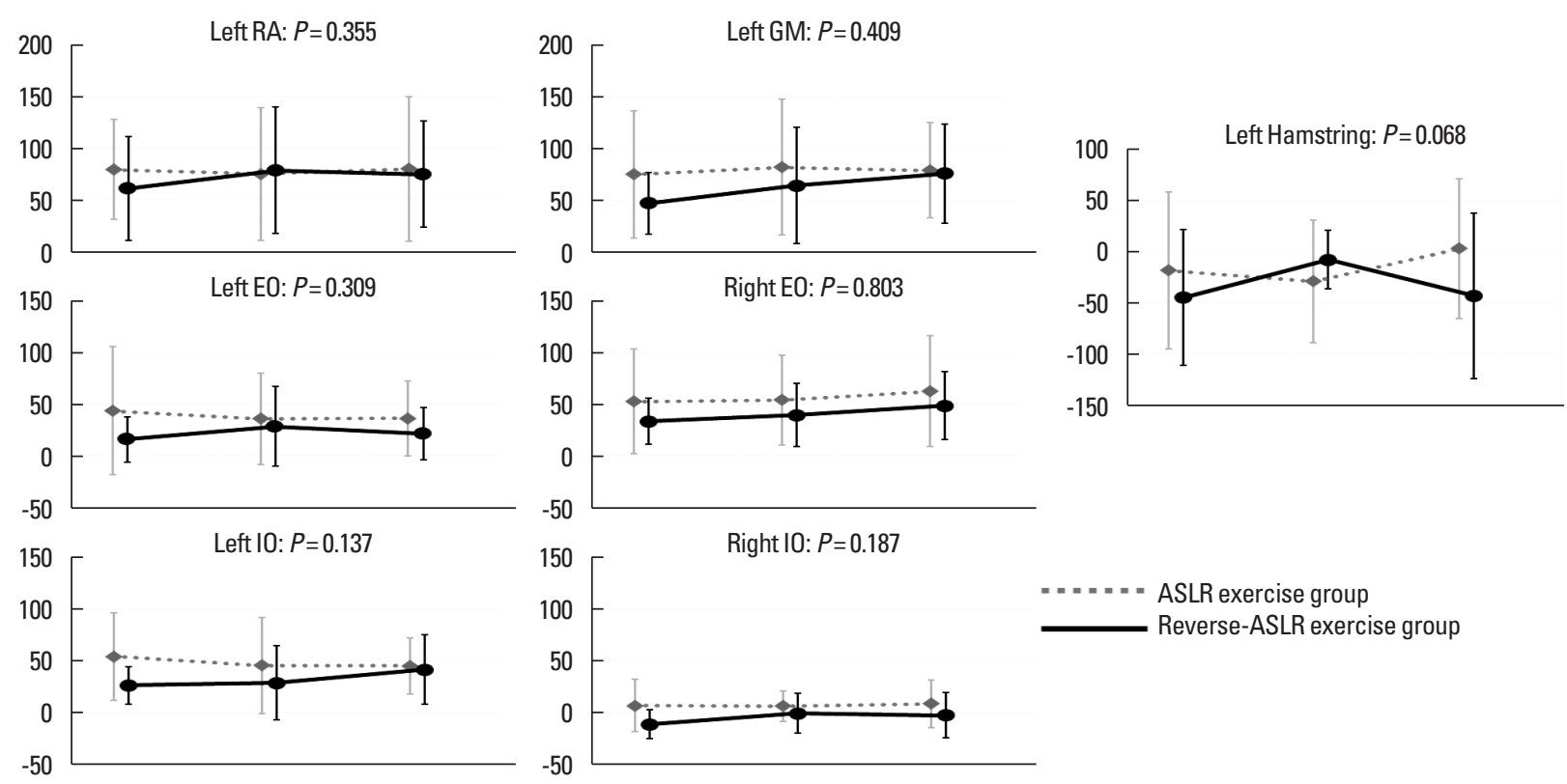

ASLR exercise group

Reverse-ASLR exercise group

Fig. 3. Relative latency of the onset of muscles to the onset of the right rectus femoris muscle during right active straight leg raising. The 3-time points on the $x$-axis represent baseline, follow-up 1, and follow-up 2. The $y$-axis represents the relative latency (msec) of the onset of each muscle to the onset of the right rectus femoris muscle during right active straight leg raising (ASLR). The positive value indicates delayed onset of the muscle to the onset of the right rectus femoris muscle during right ASLR. The negative value indicates earlier onset of the muscle to the onset of the right rectus femoris muscle during right ASLR. Means with error bars of standard deviation are presented. The $P$-value of the interaction effect is presented in each muscle. ASLR, active straight leg raising; RA, rectus abdominis; E0, external oblique; IO, internal oblique; GM, gluteus maximus.
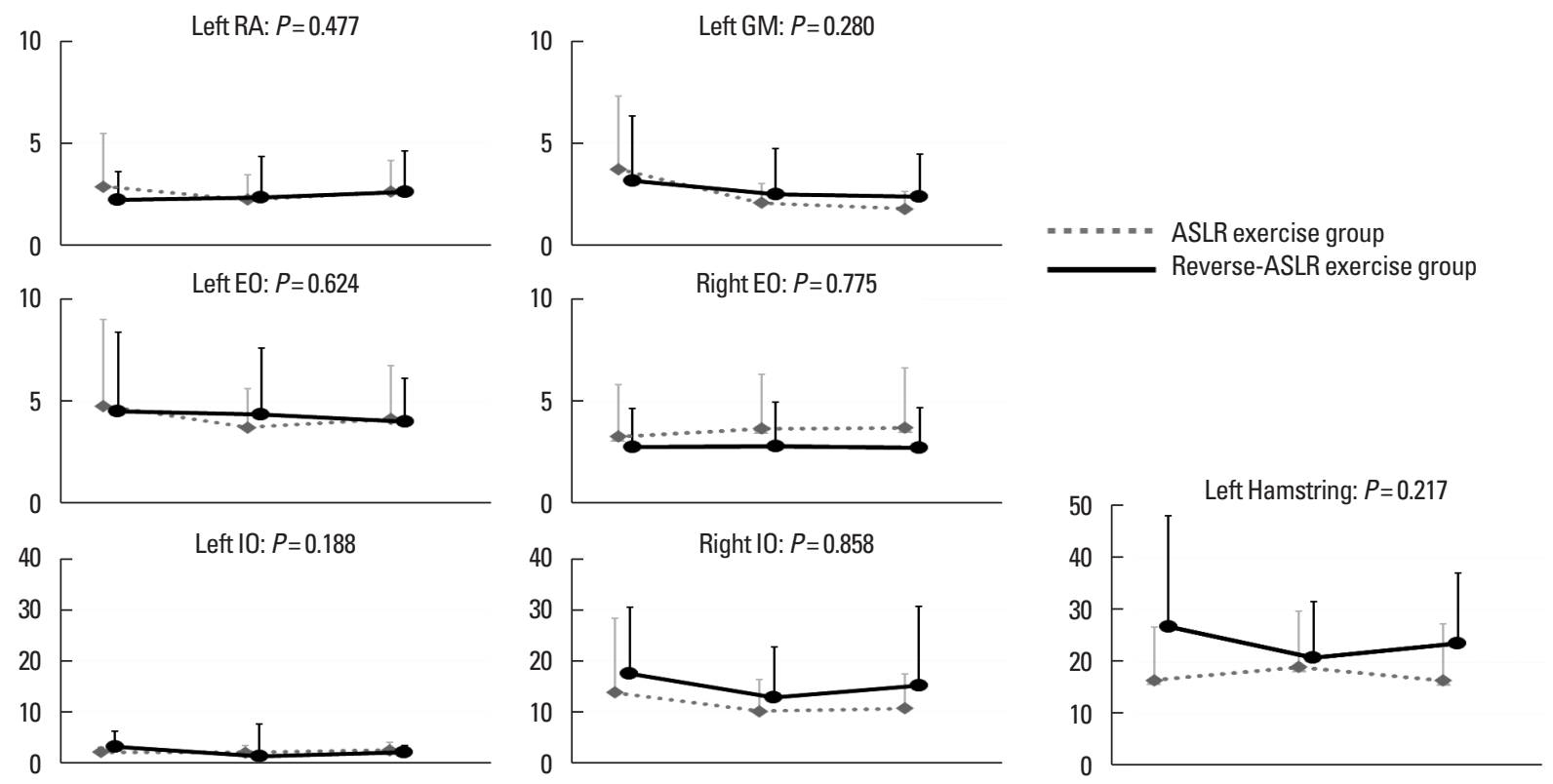

Fig. 4. Amplitude of activity in the abdominal and hip muscles for $50 \mathrm{msec}$ after the onset of the right rectus femoris muscle activity during right active strait leg raising. The 3-time points on the $x$-axis represent baseline, follow-up 1, and follow-up 2 . The $y$-axis represent the root mean square values in the $\%$ maximum voluntary contraction during the $50 \mathrm{msec}$ after the onset of the right rectus femoris muscle in right ASLR. The greater value indicates the greater amplitude of muscle activity at the early phase of the in right ASLR. Means with error bars of standard deviation are presented. The $P$-value of the interaction effect is presented in each muscle. ASLR, active straight leg raising; $R A$, rectus abdominis; EO, external oblique; IO, internal oblique; GM, gluteus maximus. 
tion effects for the measures of the 2-point discrimination test, hip abduction active repositioning acuity, and electromyography between those with and without FMS ASLR score improvement at follow-up 1. Statistically significant interaction effect was detected in the relative latency of the left rectus abdominis muscle, right

Table 2. Data of secondary outcomes at each time point except the secondary outcomes using the electromyography and time for reaching $95 \%$ of the hip flexion range

\begin{tabular}{|c|c|c|c|c|}
\hline Variable & Baseline & Follow-up 1 & Follow-up 2 & $P$-value \\
\hline \multicolumn{4}{|c|}{ Two-point discrimination testa) $(\mathrm{mm})$} & 0.336 \\
\hline ASLR & $50.36 \pm 13.23$ & $48.31 \pm 10.45$ & $48.19 \pm 10.89$ & \\
\hline Reverse-ASLR & $47.75 \pm 11.81$ & $48.75 \pm 10.24$ & $51.94 \pm 14.24$ & \\
\hline \multicolumn{4}{|c|}{ Hip abduction repositioning acuity $\left({ }^{\circ}{ }^{a}\right)$} & 0.428 \\
\hline ASLR & $4.83 \pm 3.11$ & $5.42 \pm 4.39$ & $5.62 \pm 3.64$ & \\
\hline Reverse-ASLR & $4.96 \pm 3.07$ & $4.38 \pm 4.52$ & $6.05 \pm 4.47$ & \\
\hline \multicolumn{4}{|c|}{ Euro OoL 5 Dimensions ${ }^{b)}(0-1)$} & 0.409 \\
\hline ASLR & $0.98 \pm 0.06$ & $0.98 \pm 0.06$ & $1.00 \pm 0$ & \\
\hline Reverse-ASLR & $1.00 \pm 0$ & $0.99 \pm 0.04$ & $1.00 \pm 0$ & \\
\hline
\end{tabular}

Values are presented as mean \pm standard deviation.

Positive values indicate delayed onset from the onset of rectus femoris muscle. ASLR, active straight leg raising.

alIntention-to-treat analysis, $n=20$ in each group at all time points with imputation of baseline data for missing data. ${ }^{b}$ Per-protocol analysis, $n=20$ in each group at baseline and $n=19$ in each group at follow-up 1 and follow-up 2 .
Table 4. Changes from measures at baseline to measures at follow-up 1 in participants with and without FMS ASLR score improvement to a score of 2 at follow-up 1

\begin{tabular}{|c|c|c|c|}
\hline Variable & $\begin{array}{l}\text { No improvement } \\
\quad(n=31)\end{array}$ & $\begin{array}{l}\text { Improvement } \\
(\mathrm{n}=9)\end{array}$ & $P$-value \\
\hline Relative latency of left RA (msec) & $-3.15 \pm 41.95$ & $40.74 \pm 66.44$ & 0.025 \\
\hline Relative latency of left EO (msec) & $-4.44 \pm 42.15$ & $26.97 \pm 57.33$ & 0.087 \\
\hline Relative latency of right EO (msec) & $0.06 \pm 38.75$ & $11.61 \pm 38.19$ & 0.446 \\
\hline Relative latency of left IO (msec) & $-7.93 \pm 38.25$ & $13.28 \pm 55.43$ & 0.209 \\
\hline Relative latency of right IO (msec) & $1.49 \pm 17.13$ & $17.17 \pm 18.42$ & 0.026 \\
\hline Relative latency of left GM (msec) & $8.50 \pm 55.29$ & $24.73 \pm 53.38$ & 0.451 \\
\hline Selative latency of left hamstring (msec) & $-1.54 \pm 69.98$ & $66.98 \pm 70.07$ & 0.016 \\
\hline Amplitude of activity in left RA (\%MVC) & $-0.19 \pm 2.60$ & $-0.52 \pm 1.15$ & 0.724 \\
\hline Amplitude of activity in left EO (\% MVC) & $-0.92 \pm 3.65$ & & 0.320 \\
\hline Amplitude of activity in right EO (\%MVC) & & $-0.25 \pm$ & 0.453 \\
\hline Amplitude of activity in left IO (\%MVC) & $-0.69 \pm 2.77$ & & 0.126 \\
\hline Amplitude of activity in right IO (\%MVC) & $-3.00 \pm 10.31$ & $-8.10 \pm 17.26$ & 0.444 \\
\hline Amplitude of activity in left GM (\%MVC) & $-0.82 \pm 3.01$ & $-2.31 \pm 2.33$ & 0.188 \\
\hline $\begin{array}{l}\text { Amplitude of activity in left hamstring } \\
\text { (\%MVC) }\end{array}$ & $-0.43 \pm 12.11$ & $-6.48 \pm 21.84$ & 0.302 \\
\hline Two-point discrimination test (mm) & $-1.36 \pm 12.53$ & $2.36 \pm 14.85$ & 0.468 \\
\hline Hip abduction repositioning acuity $\left({ }^{\circ}\right)$ & $0.40 \pm 4.07$ & $-1.36 \pm 3.86$ & 0.269 \\
\hline
\end{tabular}

Values are presented as mean \pm standard deviation.

ASLR, active straight leg raising; FMS, Functional Movement Screen; RA, rectus abdominis; EO, external oblique; IO, internal oblique; GM, gluteus maximus; MVC, maximum voluntary contraction.

Table 3. Data of secondary outcomes at baseline and follow-up 1 except the EQ-5D and time for reaching $95 \%$ of the hip flexion range in individuals with and without FMS ASLR score improvement to a score of 2 at follow-up 1

\begin{tabular}{|c|c|c|c|c|c|}
\hline \multirow{2}{*}{ Variable } & \multicolumn{2}{|c|}{ No improvement $(\mathrm{n}=31)$} & \multicolumn{2}{|c|}{ Improvement $(n=9)$} & \multirow{2}{*}{$P$-value } \\
\hline & Baseline & Follow-up 1 & Baseline & Follow-up 1 & \\
\hline Relative latency of left RA (msec) & $75.47 \pm 49.51$ & $72.32 \pm 44.87$ & $50.83 \pm 41.19$ & $91.57 \pm 70.08$ & 0.025 \\
\hline Relative latency of left EO (msec) & $35.49 \pm 51.54$ & $31.06 \pm 39.90$ & $10.11 \pm 17.56$ & $37.08 \pm 43.49$ & 0.215 \\
\hline Relative latency of right EO (msec) & $47.55 \pm 42.72$ & $47.61 \pm 36.77$ & $28.31 \pm 18.79$ & $39.92 \pm 38.73$ & 0.442 \\
\hline Relative latency of left IO (msec) & $47.64 \pm 35.39$ & $39.70 \pm 38.11$ & $14.16 \pm 12.64$ & $27.44 \pm 50.09$ & 0.209 \\
\hline Relative latency of right IO (msec) & $1.37 \pm 22.37$ & $2.86 \pm 16.67$ & $-15.44 \pm 13.79$ & $1.73 \pm 18.61$ & 0.026 \\
\hline Relative latency of left GM (msec) & $69.97 \pm 51.24$ & $78.47 \pm 57.32$ & $28.94 \pm 22.30$ & $53.67 \pm 65.77$ & 0.451 \\
\hline Relative latency of left hamstring (msec) & $-21.06 \pm 67.74$ & $-22.60 \pm 47.24$ & $-68.27 \pm 68.93$ & $-1.29 \pm 35.78$ & 0.016 \\
\hline Amplitude of activity in left RA (\%MVC) & $2.67 \pm 2.28$ & $2.47 \pm 1.69$ & $2.13 \pm 0.80$ & $1.61 \pm 0.98$ & 0.724 \\
\hline Amplitude of activity in left EO (\% MVC) & $4.85 \pm 4.17$ & $3.92 \pm 2.30$ & $4.04 \pm 3.04$ & $4.55 \pm 3.44$ & 0.320 \\
\hline Amplitude of activity in right EO (\%MVC) & $2.97 \pm 2.09$ & $3.33 \pm 2.28$ & $3.27 \pm 2.55$ & $3.02 \pm 2.77$ & 0.453 \\
\hline Amplitude of activity in left IO (\%MVC) & $2.75 \pm 2.50$ & $2.07 \pm 1.11$ & $2.26 \pm 1.27$ & $6.33 \pm 8.72$ & 0.009 \\
\hline Amplitude of activity in right IO (\%MVC) & $14.17 \pm 13.22$ & $11.18 \pm 7.60$ & $20.83 \pm 13.64$ & $12.73 \pm 9.79$ & 0.290 \\
\hline Amplitude of activity in left GM (\%MVC) & $3.01 \pm 3.00$ & $2.19 \pm 1.62$ & $4.87 \pm 3.86$ & $2.55 \pm 1.90$ & 0.188 \\
\hline Amplitude of activity in left hamstring (\%MVC) & $19.16 \pm 15.76$ & $18.73 \pm 10.13$ & $30.12 \pm 18.84$ & $23.64 \pm 10.52$ & 0.302 \\
\hline Two-point discrimination test (mm) & $50.11 \pm 11.65$ & $48.75 \pm 9.39$ & $45.42 \pm 13.67$ & $47.78 \pm 12.16$ & 0.468 \\
\hline Hip abduction repositioning acuity $\left({ }^{\circ}\right)$ & $4.39 \pm 2.76$ & $4.78 \pm 4.33$ & $6.65 \pm 3.16$ & $5.30 \pm 4.51$ & 0.269 \\
\hline
\end{tabular}

Values are presented as mean \pm standard deviation.

EQ-5D, Euro OoL 5 Dimensions; FMS, Functional Movement Screen; ASLR, active straight leg raising; RA, rectus abdominis; E0, external oblique; 10, internal oblique; GM, gluteus maximus; MVC, maximum voluntary contraction. 
Table 5. Data of time for reaching $95 \%$ of the hip flexion range

\begin{tabular}{lcccc} 
Group & Baseline & Follow-up 1 & Follow-up 2 & $\begin{array}{c}\text { P-value in the repeated } \\
\text { measures ANOVA }\end{array}$ \\
\hline ASLR & $0.78 \pm 0.20$ & $0.75 \pm 0.14$ & $0.74 \pm 0.14$ & 0.510 \\
Reverse-ASLR & $0.67 \pm 0.15$ & $0.71 \pm 0.15$ & $0.76 \pm 0.18$ & 0.080 \\
$P$-value in the t-test & 0.052 & 0.406 & 0.745 & \\
\hline
\end{tabular}

Values are presented as mean \pm standard deviation in sec.

ANOVA, analysis of variance; ASLR, active straight leg raising.

Per-protocol analysis was undertaken: $n=20$ in each group at baseline and $n=19$ in each group at follow-up 1 and follow-up 2.

internal oblique muscle, and left hamstring and the amplitude of the left internal oblique muscle. Table 4 presents changes in the measures of the 2-point discrimination test, hip abduction active repositioning acuity, and electromyography at baseline and follow-up 1 between individuals with and without FMS ASLR score improvement. Greater delays in the relative latency of the left rectus abdominis muscle, right internal oblique muscle, and left hamstring muscles were seen in the participants with FMS ASLR score improvement than the participants without it.

Table 5 presents time for reaching $95 \%$ of the hip flexion range in each group at each time point. No statistical difference was found neither between the groups at each time point nor between time points in each group $(P>0.05)$. Regarding the exercise compliance at follow-up 1 , no statistically significant difference $(P=$ 0.829 ) was found between the ASLR exercise group (mean \pm standard deviation, $95.49 \% \pm 7.88 \%$ ) and the reverse-ASLR exercise group $(96.01 \% \pm 6.75 \%)$.

\section{DISCUSSION}

\section{The primary outcome}

In the primary outcome, the reverse-ASLR exercise group had statistically significant improvements of the FMS ASLR score to 2, which is more than that of the ASLR exercise group. To the best of our knowledge, this is the first randomized controlled trial showing the effectiveness of the reverse patterning in exercise prescription to improve FMS scores. However, only $40 \%$ of the participants improved the FMS ASLR score to 2 with the reverseASLR exercise in 1 month. Furthermore, only half of the participants with FMS score improvement at follow-up 1 maintained the FMS score of 2 at follow-up 2. These findings indicate that further research is required to identify the optimal regime of exercise to not only improve but also maintain the FMS score.

\section{The secondary outcomes}

Considering differences between individuals with the ASLR-1SMCD and with the FMS ASLR score of 3, a hypothesis was formulated stating that the reduction of the relative latency of the onset of the right internal oblique muscle and left gluteus maximum muscle would be seen in those who had FMS ASLR score improvement (Takasaki et al., 2020). The current study was not designed to robustly investigate the hypothesis, and the findings can be based on the underpowered study design. However, interaction indicating the reduction of the relative latency of the onset of the right internal oblique muscle and left gluteus maximum muscle was not observed between the reverse-ASLR and the ASLR exercise groups and between individuals with and without FMS ASLR score improvement at follow-up 1. Furthermore, among the interaction effect analyses and changes between pre-post intervention between individuals with and without FMS ASLR score improvement at follow-up 1, findings of increased delays of the relative latency of the left rectus abdominis muscle and left hamstring muscles were consistent. A previous study (Takasaki and Okubo, 2020) demonstrated that those with ASLR-1-SMCD had further delay in the left rectus abdominis muscle during the same ASLR task with activation of the deep neck flexor muscles and indicated a potential inclusion of the deep neck flexor muscles' activation to corrective exercise. This indication may be supported by the current finding that further delay in the relative latency of the left rectus abdominis muscle may occur in those who had FMS ASLR score improvement to a score of 2. However, the delay of the relative latency in the left rectus abdominis muscle would not be a primary underlying mechanisms for the improvement of the ASLR competency because such a statistically significant difference was not seen between those with ASLR-1-SMCD and those with FMS ASLR score of 3 in a previous study (Takasaki et al., 2020). Rather, the delay in the relative latency of the left hamstring muscle in those who had FMS ASLR score improvement may provide a new hypothesis that extensor muscles in particular global muscles may play important roles as underlying mechanisms for the improvement of the ASLR competency. Thus, further studies focusing on the extensor muscles are required.

Additionally, this study preliminarily investigated the effect of the exercises on sensorimotor control measures, including the two-point discrimination test and the active repositioning test of the right hip in the secondary outcomes. Neither interaction nor changes in the post hoc analysis were observed. Like the relative latency, while the results of the secondary outcomes can be based on the underpowered study design, these findings indicate that the 
reverse-ASLR and the ASLR exercises are not promising exercise regimes and specific exercises are required to change the sensorimotor control measures.

\section{Limitations}

This study had two potential limitations. First, this randomized controlled trial used convenience sampling, and the participants were young, limiting generalizability of the findings. Second is associated with the limited abdominal muscles assessed using the electromyography, which is simply due to a technical limitation. Comprehensive evaluation of the abdominal muscles will be required to identify key motor control impairments among individuals with ASLR-1-SMCD, including using a fine-wire electromyography for the transversus abdominis muscle. However, a cross-talk was present between the internal oblique muscle and the transversus abdominis muscle on the surface electromyography (McGill et al., 1996), and findings in the relative latency of the internal oblique muscles were inconsistent among the analyses by categorizing those with and without FMS ASLR score improvement at follow-up 1 and the analysis comparing those with ASLR-1-SMCD and those with FMS ASLR score of 3 in a previous study (Takasaki et al., 2020). Therefore, the transversus abdominis muscle is believed to be the muscle of less priority to further investigate as underlying mechanisms for the improvement of the FMS ASLR score.

In conclusion, the results of the primary outcome of this study indicated that the reverse-ASLR exercise was more effective than the repeating ASLR exercise in improving the FMS ASLR score among individuals with ASLR-1-SMCD. This study provided a foundation for future studies to identify an ideal corrective exercise to improve the ASLR score among individuals with ASLR-1SMCD and underlying mechanisms of the ASLR-1-SMCD.

\section{CONFLICT OF INTEREST}

No potential conflict of interest relevant to this article was reported.

\section{ACKNOWLEDGMENTS}

The authors received no financial support for this article.

\section{REFERENCES}

Arvin M, Hoozemans MJ, Burger BJ, Verschueren SM, van Dieen JH, Pijnappels M. Reproducibility of a knee and hip proprioception test in healthy older adults. Aging Clin Exp Res 2015;27:171-177.

Bennett H, Davison K, Arnold J, Slattery F, Martin M, Norton K. Multicomponent musculoskeletal movement assessment tools: a systematic review and critical appraisal of their development and applicability to professional practice. J Strength Cond Res 2017;31:2903-2919.

Cook G. Movement: Functional movement systems: screening, assessment, corrective strategies. Santa Cruz: On Target Publications; 2010.

Criswell E. Cram's introduction to surface electromyography. 2nd ed. Sudbury: Jones and Bartlett Publishers; 2011.

Ehrenbrusthoff K, Ryan CG, Grüneberg C, Martin DJ. A systematic review and meta-analysis of the reliability and validity of sensorimotor measurement instruments in people with chronic low back pain. Musculoskelet Sci Pract 2018;35:73-83.

Ehrenbrusthoff K, Ryan CG, Grüneberg C, Wolf U, Krenz D, Atkinson G, Martin DJ. The intra- and inter-observer reliability of a novel protocol for two-point discrimination in individuals with chronic low back pain. Physiol Meas 2016;37:1074-1088.

Faul F, Erdfelder E, Lang AG, Buchner A. G*Power 3: a flexible statistical power analysis program for the social, behavioral, and biomedical sciences. Behav Res Methods 2007;39:175-191.

Hodges PW, Bui BH. A comparison of computer-based methods for the determination of onset of muscle contraction using electromyography. Electroencephalogr Clin Neurophysiol 1996;101:511-519.

Jull G, Falla D, Treleaven J, Hodges P, Vicenzino B. Retraining cervical joint position sense: the effect of two exercise regimes. J Orthop Res 2007;25:404-412.

Lotze M, Moseley GL. Role of distorted body image in pain. Curr Rheumatol Rep 2007;9:488-496.

McGill S, Juker D, Kropf P. Appropriately placed surface EMG electrodes reflect deep muscle activity (psoas, quadratus lumborum, abdominal wall) in the lumbar spine. J Biomech 1996;29:1503-1507.

Mirdamadi JL, Block HJ. Somatosensory changes associated with motor skill learning. J Neurophysiol 2020;123:1052-1062.

Moran RW, Schneiders AG, Major KM, Sullivan SJ. How reliable are Functional Movement Screening scores? A systematic review of rater reliability. Br J Sports Med 2016;50: 527-536.

Nishimoto K, Takasaki H. Jack-knife stretching and active knee extension stretching equally improve the relative flexibility of the hamstring muscles between the low back: a randomized controlled trial. Phys Ther Sport 2019;38:139-145.

Ostry DJ, Darainy M, Mattar AA, Wong J, Gribble PL. Somatosensory plasticity and motor learning. J Neurosci 2010;30:5384-5393.

Ostry DJ, Gribble PL. Sensory plasticity in human motor learning. Trends Neurosci 2016; 39:114-123.

Sandvik L, Erikssen J, Mowinckel P, Rodland EA. A method for determin- 
ing the size of internal pilot studies. Stat Med 1996;15:1587-1590.

Shiroiwa T, Ikeda S, Noto S, Igarashi A, Fukuda T, Saito S, Shimozuma K. Comparison of value set based on DCE and/or TTO data: scoring for EQ-5D-5L health states in Japan. Value Health 2016;19:648-654.

Takasaki H, Aoki S, May S. No increase in 6-week treatment effect of Mechanical Diagnosis and Therapy with the use of the LUMOback in people with non-acute non-specific low back pain and a directional preference of extension: a pilot randomized controlled trial. Physiotherapy 2018;104:347-353.

Takasaki H, Kawazoe S, Wahara T, Goto A. Earlier onsets in internal oblique and gluteus maximus muscles during leg raising in Functional Movement Screen score 3 than score 1. J Exerc Rehabil 2020;25:363-368.

Takasaki H, Okubo Y. Deep neck flexors impact rectus abdominal muscle in active straight leg raising. Int J Sports Phys Ther 2020;15:1044-1051.

Tejani AS, Middleton EF, Huang M, Dimeff RJ. Implementing a standardized interventional exercise regimen to improve functional movements in female collegiate athletes. Int J Sports Phys Ther 2019;14:117-126.

Warren M, Lininger MR, Chimera NJ, Smith CA. Utility of FMS to understand injury incidence in sports: current perspectives. Open Access J Sports Med 2018;9:171-182.

Wattananon P, Klomjai W, Sung W. One session of motor control exercise improves joint position sense assessed by an iPhone application: a randomized controlled trial. J Phys Ther Sci 2019;31:583-589.

Wong JD, Wilson ET, Gribble PL. Spatially selective enhancement of proprioceptive acuity following motor learning. J Neurophysiol 2011;105: 2512-2521. 